\title{
Case Report \\ Canine Choroid Plexus Tumor with Intracranial Dissemination Presenting as Multiple Cystic Lesions
}

\author{
Trisha J. Oura, ${ }^{1}$ Peter J. Early, ${ }^{2}$ Samuel H. Jennings, ${ }^{2}$ Melissa J. Lewis, ${ }^{2}$ \\ Jeremy R. Tobias, ${ }^{2}$ and Donald E. Thrall ${ }^{3}$ \\ ${ }^{1}$ Tufts Veterinary Emergency Treatment and Specialties, 525 South Street, Walpole, MA 02081, USA \\ ${ }^{2}$ North Carolina State University Veterinary Teaching Hospital, 1052 William Moore Drive, Raleigh, NC 27607, USA \\ ${ }^{3}$ Associate Dean for Research, Ross University School of Veterinary Medicine, P.O. Box 334, Basseterre, Saint Kitts, USA \\ Correspondence should be addressed to Peter J. Early; pjearly@ncsu.edu
}

Received 9 April 2013; Accepted 9 May 2013

Academic Editors: S. C. Rahal, M. Santos, R. M. Santos, and S. Stuen

Copyright (C) 2013 Trisha J. Oura et al. This is an open access article distributed under the Creative Commons Attribution License, which permits unrestricted use, distribution, and reproduction in any medium, provided the original work is properly cited.

\begin{abstract}
A Miniature Pinscher developed acute blindness and behavioral changes. On magnetic resonance imaging (MRI), there were multiple small intra-axial cystic lesions, and primary differential diagnoses included primary or metastatic neoplasia and neurocysticercosis. These cystic lesions were subsequently diagnosed histopathologically as disseminated choroid plexus carcinoma. This is only the second documented description of this diagnosis in a dog, but both patients had very similar MRI findings. This patient adds to the literature about the MRI characteristics of choroid plexus tumors and indicates that choroid plexus tumor should be considered as a possible cause of small multifocal intra-axial cystic brain lesions in dogs, regardless of whether a primary intraventricular lesion is visible.
\end{abstract}

\section{Introduction}

Choroid plexus tumors arise from the epithelium of the choroid plexus and account for approximately $10 \%$ of all primary intracranial tumors in dogs [1]. These predominantly benign tumors are found most commonly in the lateral, third, or fourth ventricles. Dogs are typically middle-aged at the time of presentation, and males and Golden Retrievers may be overrepresented [1]. Clinical signs, which can include ataxia, circling, blindness, and behavior changes, are often due to hydrocephalus as ventricular lesions may cause cerebrospinal fluid (CSF) accumulation due to obstruction and/or overproduction $[1,2]$.

Definitive diagnosis of choroid plexus tumors is by histopathologic evaluation; however, there are often distinctive findings on magnetic resonance imaging (MRI), such as well-differentiated, irregularly margined, intraventricular masses. These masses are typically hyperintense on $\mathrm{T} 2$ and proton density (PD) sequences with strong, homogeneous contrast enhancement [3]. Perilesional edema may be present or absent, and primary choroid plexus tumors may contain foci of hemorrhage or mineralization [3].
Both choroid plexus papillomas and carcinomas can metastasize, often to the spinal subarachnoid space, creating the so-called drop metastases. Rarely, tumor dissemination and meningeal carcinomatosis of choroid plexus tumors have been documented, with spread of neoplastic cells within the leptomeninges $[4,5]$. Although documented histologically [5], there has been only one prior report of the MRI findings of a dog with choroid plexus carcinoma and secondary leptomeningeal metastases $[4,5]$. The purpose of this report is to add information from one additional patient with this interesting and unusual manifestation of choroid plexus tumor.

\section{Case Presentation}

A 9-year-old neutered male Miniature Pinscher developed acute-onset blindness and possible seizure activity. When examined, the patient was anxious, vocalizing, and exhibiting compulsive behaviors. Both pupils were mydriatic with absent menace response, absent dazzle, and absent direct and indirect papillary light reflexes. Mild to moderate cervical pain was elicited on palpation and during range of motion. 


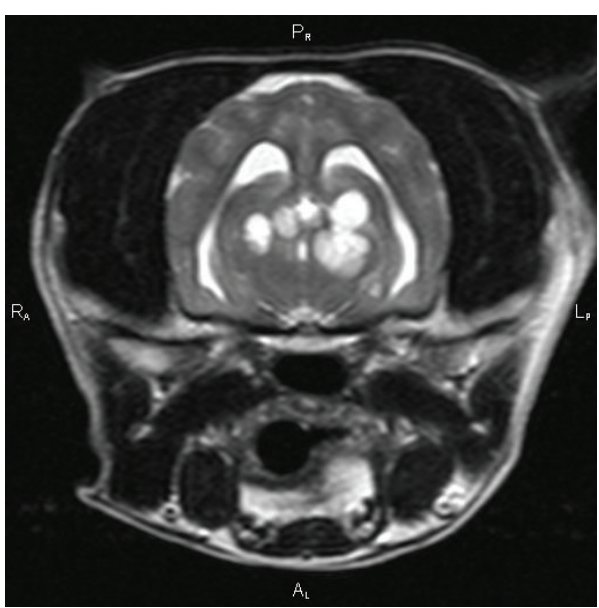

(a)

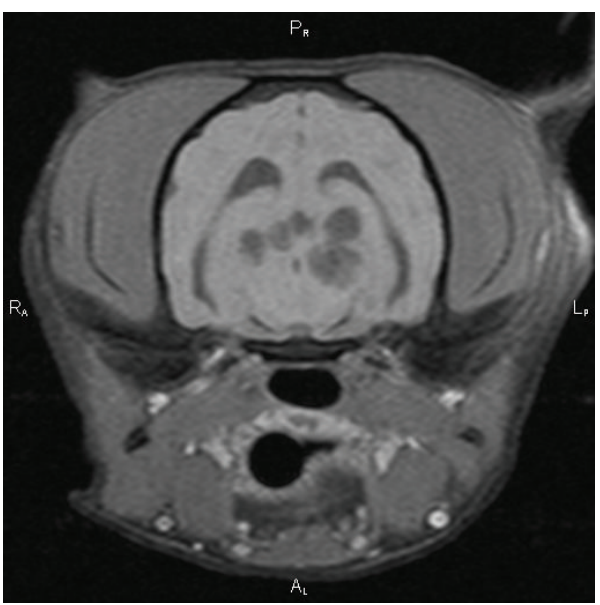

(c)

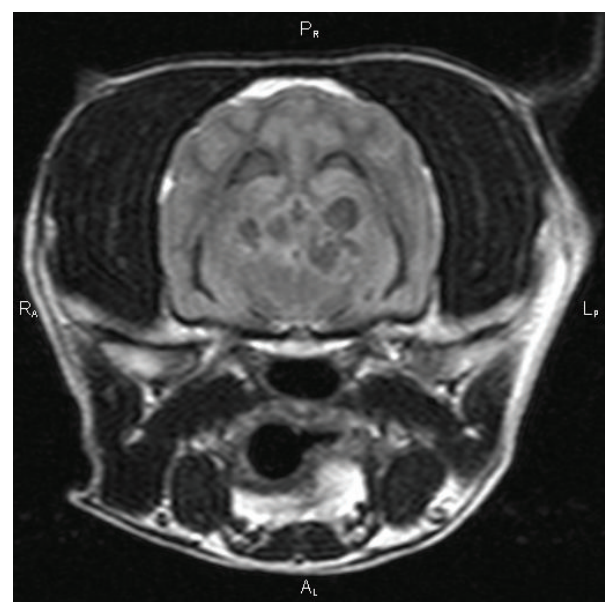

(b)

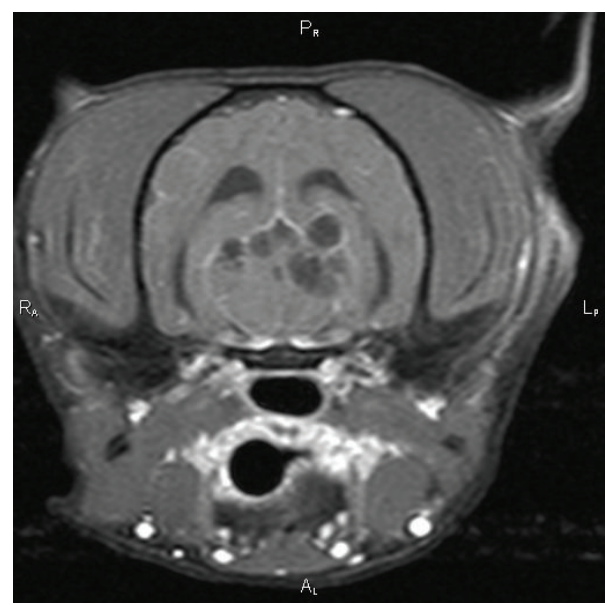

(d)

Figure 1: Transverse magnetic resonance images (MRIs) at the level of the mesencephalic aqueduct. (a) T2W image (TE/TR, 104/4440 ms) with several round lesions within the caudal thalamus that are isoattenuating to cerebrospinal fluid within the lateral ventricles. (b) These lesions null on FLAIR sequences (TR/TE, 78/9000 ms) with mild perilesional hyperintensities representing edema. (c) The lesions are isoattenuating to CSF on T1W images (TE/TR, 12/53 ms) and have mild contrast enhancement of the lesion wall (d).

The neuroanatomic localization was multifocal involving the prosencephalon and brainstem and or possible cervical involvement.

Magnetic resonance imaging of the brain was performed using a 1.5 T magnet (Symphony $1.5 \mathrm{~T}$; Siemens Medical Solutions USA, Inc., Malvern, PA, USA). Caudal to the interthalamic adhesion, there were numerous small, $\sim 1.0-10.0 \mathrm{~mm}$ diameter, intra-axial, thin-walled, T2 hyperintense (TE/TR, 104/4440 ms), T1-hypointense (TE/TR, 12/53 ms) nodules within the thalamus, colliculi, and hippocampus. The T2signal of these nodules was nulled in FLAIR images (TR/TE, $78 / 9000 \mathrm{~ms}$ ) indicating a cystic nature, and the wall of the nodules enhanced following intravenous administration of gadoversetamide contrast medium (Optimark; Mallinkrodt Inc., St. Louis, MO, USA). Based on the FLAIR sequences, there was also mild perilesional edema (Figure 1). There was mild obstructive hydrocephalus due to mesencephalic aqueduct compression by the more caudal nodules, mild transtentorial herniation causing compression of the rostral aspect of the cerebellum, and syrinx formation in the cranial aspect of the cervical spinal cord. Additional smaller nodules were present in the ventral aspect of the cerebellum. Based on the MRI findings, differential diagnoses for the cystic lesions included atypical cystic metastatic neoplasia or neural cysticercosis, though the latter was considered much less likely as the animal was not known to have lived in or travelled to an area endemic for cysticercosis.

Differential diagnoses for the cystic lesions included atypical cystic metastatic neoplasia or neural cysticercosis. Obstructive hydrocephalus and compression of the lateral geniculate nuclei by the cystic lesions were thought to be possible causes of the blindness.

On CSF analysis, there was a mild mononuclear pleocytosis $(21$ cells/uL) and an elevated total protein $(54.5 \mathrm{mg} / \mathrm{dL})$. No etiologic agents or neoplastic cells were identified.

The patient was discharged with prednisone $5 \mathrm{mg}$ tablet given orally once every 24 hours, omeprazole $10 \mathrm{mg}$ capsule given orally once every 24 hours, aspirin $5 \mathrm{mg}$ capsule given 
orally once every 24 hours, enalapril $3.75 \mathrm{mg}$ tablet orally given every 12 hours, albendazole $227 \mathrm{mg}$ oral suspension given orally every 12 hours for 3 days, and praziquantel/ pyrantel pamoate/febantel $54.4 \mathrm{mg}$ tablet given orally once.

Within four days of discharge, the patient had an improved mental status with decreased compulsive behavior. However, within one week the patient exhibited generalized seizure activity, and anticonvulsant therapy was initiated. Approximately three weeks after discharge, the patient developed cluster seizures and was euthanized.

At postmortem examination, the cystic lesions and mild hydrocephalus were confirmed. There were also several additional pinpoint to $0.1 \mathrm{~cm}$ cystic foci within the cerebral leptomeninges (Figure 2). On histopathology, approximately $20 \%$ of the left thalamus was replaced by scattered, variably sized, well-demarcated, expansile, unencapsulated, cystic intraventricular, and intraparenchymal neoplasms. Most of the clear cystic cavities were often lined by a relatively uniform population of cuboidal to columnar cells arranged in a single layer with a few, simple to complex, intraluminal papillary projections supported by thin fibrovascular cores. These cells had well-defined cell borders with small to moderate amount of eosinophilic cytoplasm with an oval, basal nucleus with finely stippled chromatin and an indistinct nucleolus. The cells exhibited minimal anisocytosis and anisokaryosis. Occasionally, however, the cysts and papillary projections were lined by up to eight layers of jumbled epithelial cells with more variably sized, shaped, and located nuclei with more open chromatin. There was rare single cell necrosis within the neoplastic population. The mitotic rate varied regionally from zero to seven mitotic figures in 10 high magnification (400x) fields. The cysts occasionally contained small amounts of laminated, mineralized material. The adjacent neuropil was compressed and vacuolated with mild gliosis and, rarely, a few perivascular lymphocytes and plasma cells. Similar cystic neoplasms were present within sections of brainstem bilaterally adjacent to the fourth ventricle, presumed to be in the lateral apertures, and throughout the cerebral and cerebellar meninges. The final diagnosis was choroid plexus carcinoma with multifocal intracranial metastasis and mild, secondary hydrocephalus.

\section{Discussion}

Presently there is only one other description of the MRI findings associated with intracranial dissemination of a choroid plexus tumor in a dog. Given the rarity of this condition, it is important that additional patients be described as they are discovered. Similar clinical finding between both dogs included blindness and behavioral changes. On MRI, both patients had mild hydrocephalus and similar sized intra-axial cystic lesions which, in our patient, were located primarily in the caudal thalamus, colliculi and hippocampus, while in the previously reported patient, additional lesions were also found throughout the temporal and frontal lobes [4]. The cystic lesions in our patient were also associated with mild perilesional edema and mild contrast enhancement of the cyst walls which was not observed previously. This difference

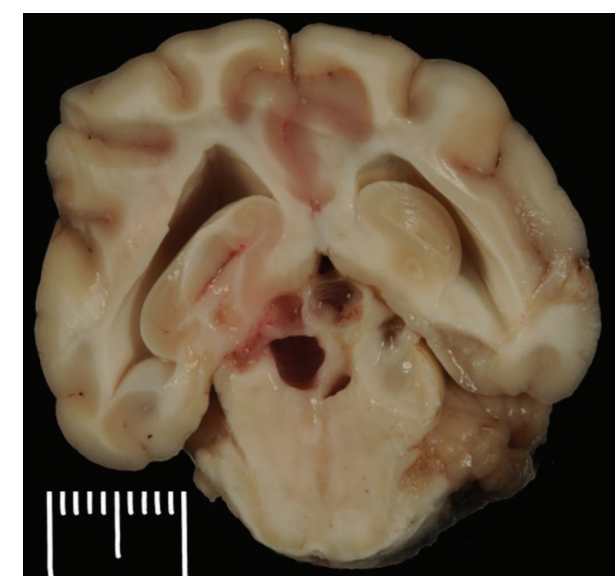

Figure 2: Cross-section of the dog's brain at the level of the mesencephalic aqueduct. Several 2-3 mm, round to oval, cystic lesions multifocally expand and replace the dorsal thalamic parenchyma and compress the mesencephalic aqueduct. Histopathologically, these corresponded to choroid plexus tumors.

may be due to the fact that the previous patient received glucocorticoids prior to MRI acquisition [4]. The primary tumor could not be identified in either patient.

Meningeal carcinomatosis is uncommon in animals but has been documented with metastatic mammary carcinoma and carcinoma without an identified primary tumor [6, 7]. Specifically, intraparenchymal dissemination of choroid plexus tumors is documented rarely $[4,5]$. Intracranial dissemination of choroid plexus tumors is also uncommon in people, with few reports of neoplasia involving the leptomeninges or seeding of the subarachnoid space [8-10]. One human patient had multiple, nonenhancing, cystic subarachnoid lesions of the cerebellum, brainstem, and frontal lobe on MRI, an appearance very similar to our findings [9].

\section{Conclusion}

Multiple intra-axial cystic lesions are an unusual manifestation of choroid plexus tumors in both people and animals. More commonly, choroid plexus tumors are typically solitary, lobulated, strongly contrast-enhancing lesions within the ventricular system [3]. This case report of a canine patient adds to the available literature regarding choroid plexus tumors and indicates that disseminated choroid plexus tumor should be considered as a differential diagnosis for multiple small intra-axial cysts in dogs, regardless of whether a primary intraventricular tumor is visible.

\section{References}

[1] D. R. Westworth, P. J. Dickinson, W. Vernau et al., "Choroid plexus tumors in 56 dogs (1985-2007)," Journal of Veterinary Internal Medicine, vol. 22, no. 5, pp. 1157-1165, 2008.

[2] F. A. Zaki and L. A. Nafe, "Choroid plexus tumors in the dog," Journal of the American Veterinary Medical Association, vol. 176, no. 4, pp. 328-330, 1980. 
[3] S. L. Kraft, P. R. Gavin, C. DeHaan, M. Moore, L. R. Wendling, and C. W. Leathers, "Retrospective review of 50 canine intracranial tumors evaluated by magnetic resonance imaging," Journal of Veterinary Internal Medicine, vol. 11, no. 4, pp. 218-225, 1997.

[4] D. Lipsitz, R. E. Levitski, and A. E. Chauvet, "Magnetic resonance imaging of a choroid plexus carcinoma and meningeal carcinomatosis in a dog," Veterinary Radiology and Ultrasound, vol. 40, no. 3, pp. 246-250, 1999.

[5] A. K. Patnaik, R. A. Erlandson, and P. H. Lieberman, "Choroid plexus carcinoma with meningeal carcinomatosis in a dog," Veterinary Pathology, vol. 17, no. 3, pp. 381-385, 1980.

[6] M. Pumarola and M. Balasch, "Meningeal carcinomatosis in a dog," Veterinary Record, vol. 138, no. 21, pp. 523-524, 1996.

[7] I. Mateo, V. Lorenzo, A. Muñoz, and J. Molín, "Meningeal carcinomatosis in a dog: magnetic resonance imaging features and pathological correlation," Journal of Small Animal Practice, vol. 51, no. 1, pp. 43-48, 2010.

[8] R. Leblanc, S. Bekhor, D. Melanson, and S. Carpenter, "Diffuse craniospinal seeding from a benign fourth ventricle choroid plexus papilloma. Case report," Journal of Neurosurgery, vol. 88, no. 4, pp. 757-760, 1998.

[9] T. McCall, M. Binning, D. T. Blumenthal, and R. L. Jensen, "Variations of disseminated choroid plexus papilloma: 2 case reports and a review of the literature," Surgical Neurology, vol. 66, no. 1, pp. 62-67, 2006.

[10] F. Doglietto, L. Lauretti, T. Tartaglione, M. Gessi, E. Fernandez, and G. Maira, "Diffuse craniospinal choroid plexus papilloma with involvement of both cerebellopontine angles," Neurology, vol. 65, no. 6, p. 842, 2005. 

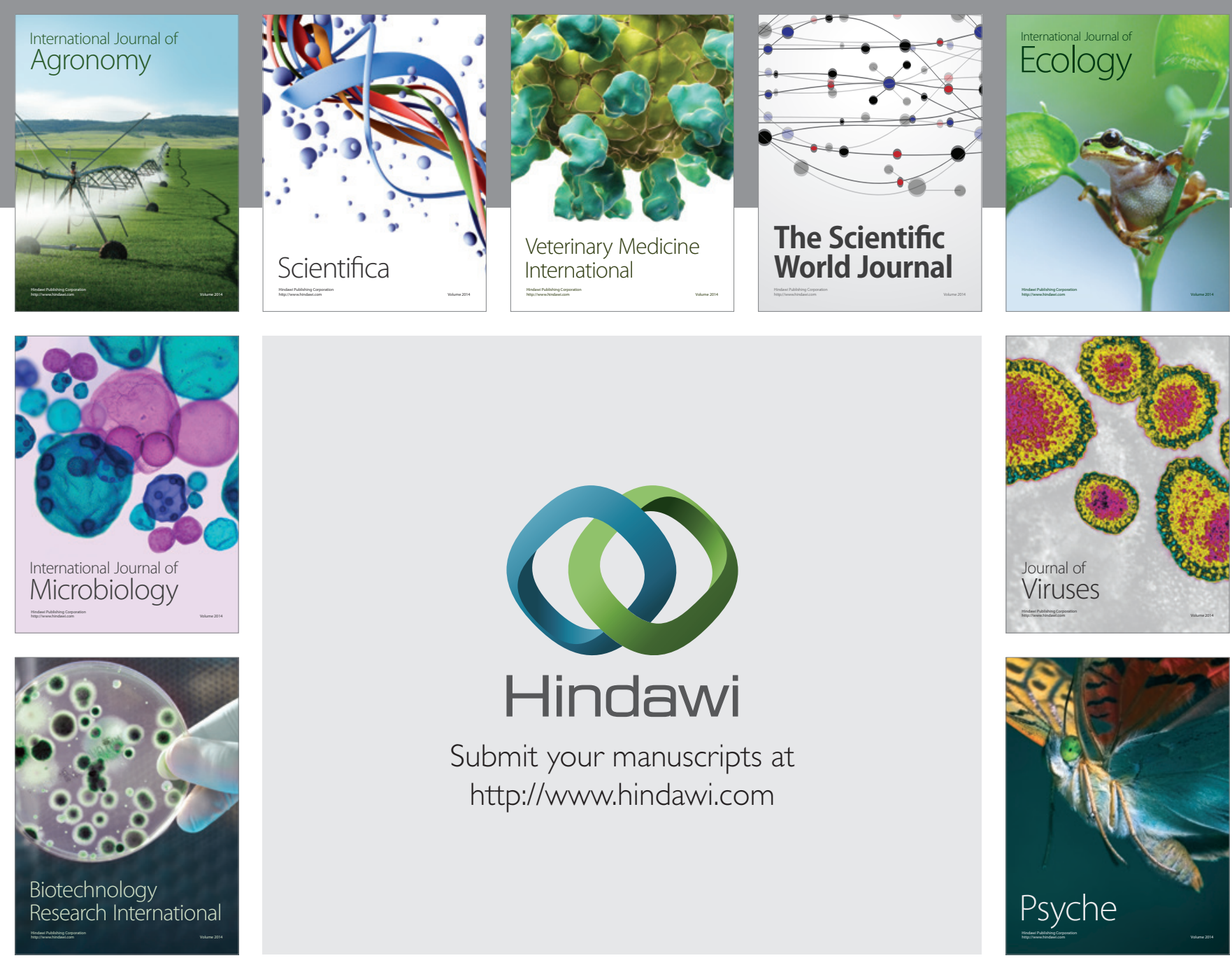

Submit your manuscripts at http://www.hindawi.com
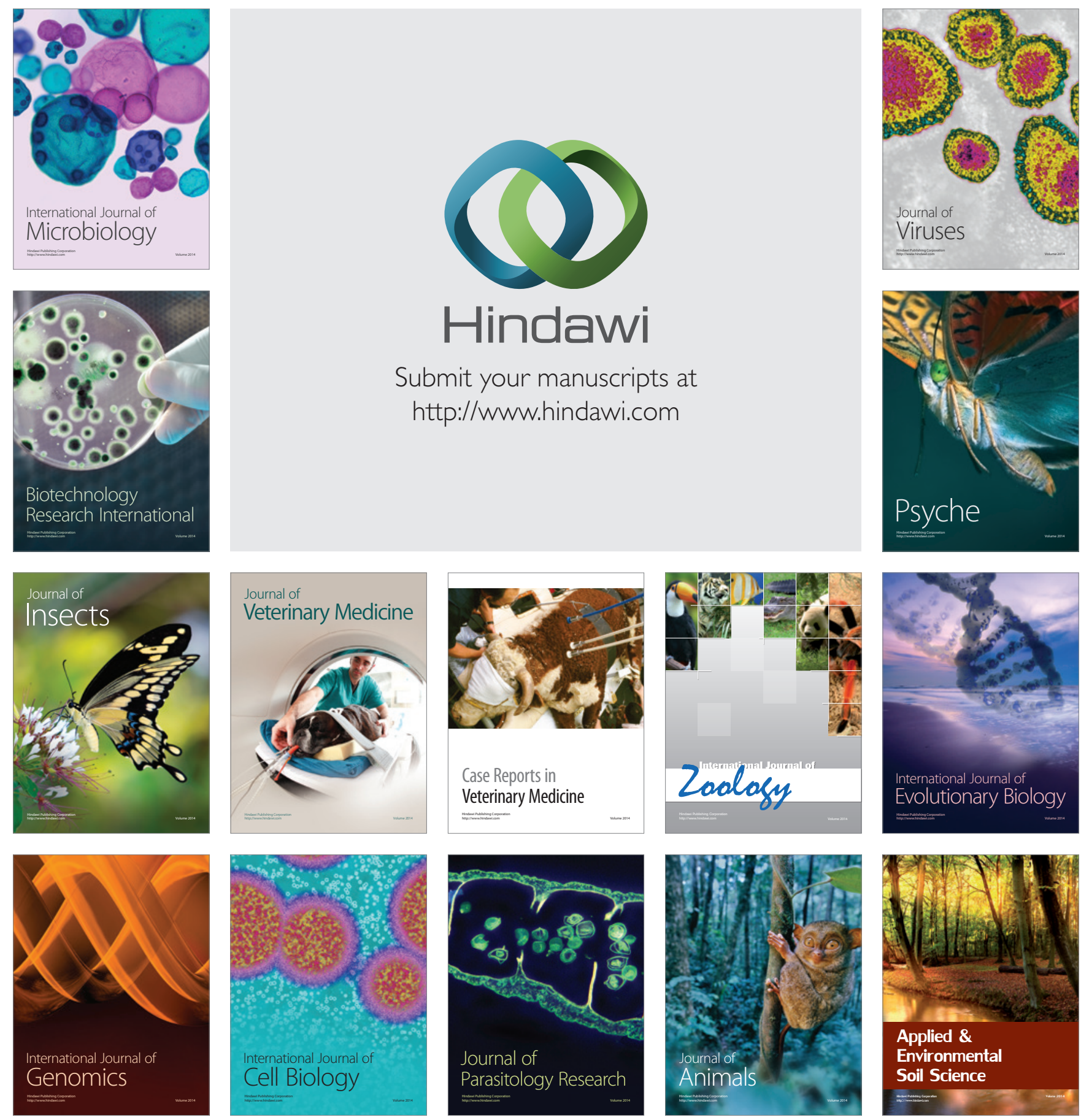